\title{
EFFECT OF PERMANENT-MAGNET IRREGULARITIES IN LEVITATION FORCE MEASUREMENTS*
}

\author{
John R. Hull \\ Energy Technology Division, Argonne National Laboratory \\ 9700 South Cass Avenue, Argonne, Illinois 60439
}

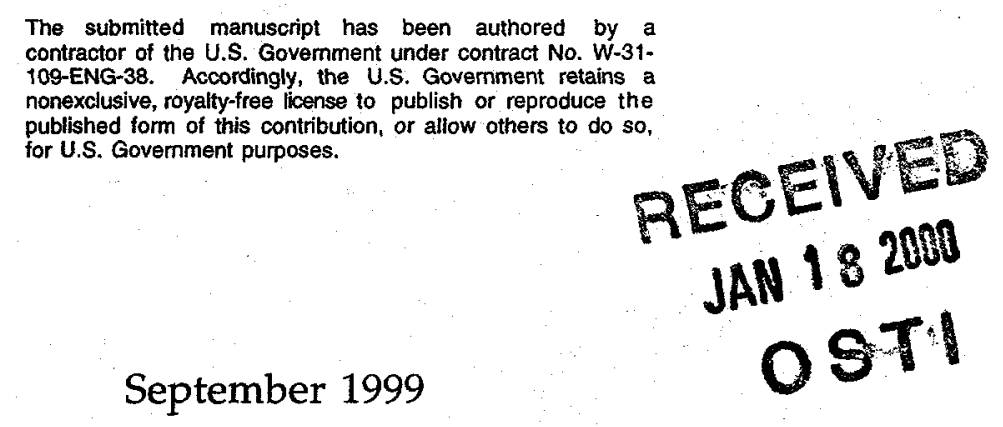

To be presented at 2nd International Workshop on the Processing and Applications of Superconducting (RE)BCO Large Grain Materials,

Morioka, Japan, Oct. 20-22, 1999.

Workshop papers to be published as journal articles in Superconductor Science and Technology.

${ }^{*}$ Work supported by the U.S. Department of Energy, Energy Efficiency and Renewable Energy, as part of a program to develop electric power technology, under Contract W-31-109-Eng-38. 


\section{DISCLAIMER}

This report was prepared as an account of work sponsored by an agency of the United States Government. Neither the United States Government nor any agency thereof, nor any of their employees, make any warranty, express or implied, or assumes any legal liability or responsibility for the accuracy, completeness, or usefulness of any information, apparatus, product, or process disclosed, or represents that its use would not infringe privately owned rights. Reference herein to any specific commercial product, process, or service by trade name, trademark, manufacturer, or otherwise does not necessarily constitute or imply its endorsement, recommendation, or favoring by the United States Government or any agency thereof. The views and opinions of authors expressed herein do not necessarily state or reflect those of the United States Government or any agency thereof. 


\section{DISCLAIMER}

Portions of this document may be illegible in electronic image products. Images are produced from the best available original document. 


\title{
EFFECT OF PERMANENT-MAGNET IRREGULARITIES IN LEVITATION FORCE MEASUREMENTS
}

\author{
John R. Hull \\ Energy Technology Division \\ Argonne National Laboratory, Argonne, IL 60439 USA
}

In the measurement of the levitation force between a vertically magnetized permanent magnet (PM) and a bulk high-temperature superconductor (HTS), PM domains with horizontal components of magnetization are shown to produce a nonnegligible contribution to the levitation force in most systems. Such domains are typically found in all PMs, even in those that exhibit zero net horizontal magnetic moment. Extension of this analysis leads to an HTS analog of Earnshaw's theorem, in which at the field-cooling position the vertical stiffness is equal to the sum of the horizontal stiffnesses, independent of angular distribution of magnetic moments within the PM.

\section{INTRODUCTION}

Measurement of the levitation force between a permanent magnet (PM) and a bulk hightemperature superconductor (HTS) has become a popular method to characterize HTSs. A number of PM properties contribute to its utility in these measurements for the study of (RE)BCO HTSs. The measurement is directly applicable to the design of HTS bearings, which rely on the levitation force for their basic operation [1]. SmCo or FeBNd PMs have internal magnetizations $\mu_{\mathrm{o}} \mathrm{M}>1 \mathrm{~T}$, which is usually sufficient to determine the penetration magnetic field and other performance parameters of the HTSs. These PMs are commecially available in sizes commensurate with the size of single-domain (RE)BCO. The PMs are compatible with submergence in liquid nitrogen (LN) and do not need a power supply. The magnetizations are 
steady in time at a fixed temperature.

Sophisticated analytical and numerical models that are based on the properties of the PM and the HTS have been developed to predict the levitation force in axially symmetric systems [2-6]. Typically, when predictions of these models are compared with experimental results, the PM is assumed to be magnetized in the axial direction.

The present manufacture of high energy-product PMs involves aligning the magnetic powder in a magnetic field to orient the easy axis of magnetization [7]. This green compact is then sintered to obtain final density. Under typical alignment procedures, there is a substantial self demagnetization effect, and cylindrical compacts that are otherwise very homogenous will have an alignment distribution that is concave. Locally, this alignment configuration gives rise horizontal components to the magnetic moment. Globally, there is no net horizontal moment in the ideal case, although the vertical moment is reduced from the theoretical maximum. Imperfections in the manufacturing process further exacerbate this situation and may give rise to net horizontal moments. Typical mean angular misorientations of grains in PMs such as the ones we are using are in the range of $20-40^{\circ}[8,9]$.

In this paper I discuss the effect of horizontal magnetic moments in PM/HTS levitation measurements. I show that ignoring their presence can lead to erroneous predictions in which the measured levitation force may even exceed the theoretical maximum between the PM and its diamagnetic image. The theoretical maximum force between a PM and its image has been suggested for an absolute standard in comparing the performance of an HTS in a PM/HTS system. Thus, it is important to understand the scope of influence of the horizontal moment on the calculation. In this paper I use the frozen-flux model $[10,11]$, which provides an economy in understanding the relevant features of the levitation forces and stiffnesses in this case but does not predict hysteresis loss or account for the physics of the more physically correct critical-state model. 


\section{FROZEN-FLUX MODEL}

The general schematic of the frozen-flux model for a dipole-dipole interaction is shown in Fig. 1 , in which the HTS surface is a horizontal plan. In zero-field cooling (ZFC), the PM produces its diamagnetic mirror image below the HTS top surface, while in field cooling $(\mathrm{FC})$, two images appear: one is the diamagnetic mirror image and the other is the frozen image, as shown in Fig. 1(a). The interaction force between PM and HTS is provided by the superposition of the magnetic field from the PM, the source of the frozen image (due to trapped flux in the HTS), and the diamagnetic mirror image (due to screening currents in the HTS). The diamagnetic mirror image moves when the PM moves so that its lateral position equals that of the PM and its vertical height below the HTS surface equals the height of the PM above the surface. Once formed, the frozen image does not move, and has coordinates $(0,-\mathrm{h})$. The magnitude of the magnetic moment of the frozen image is exactly equal to that of the PM so that there is no net force upon field cooling. This is the typical case with good melt-textured HTSs, such as those that we are using, in which the Meissner fraction after field cooling is negligibly small.

An advantage of the frozen-flux model in this discussion is that there are no free parameters. The forces may be derived from measured properties of the PM. For a first-order approximation, the PM/HTS interaction can be represented by the forces between dipole images. This approximation readily shows the basic concept of the frozen-image model and produces readily derived analytical expressions. Calculation of forces in realistic geometries requires numerical integration over three dimensions in the most general case. Figure 1(b) shows how to determine the image moments if the PM dipole is oriented at angle $\theta_{1}$ to the vertical. The orientation angle $\theta_{2}$ for the diamagnetic image is given by $\theta_{2}=\pi-\theta_{1}$. The angle for the frozen image is given by $\theta_{2}=-\theta_{1}$. The magnitude of the image moments are identical to that of the PM moment. 
A physically equivalent, but numerically easier, method to calculate PM/HTS forces in cylindrical geometries is to model the PM and its images by equivalent Amperian current loops [11]. In the most general case, a two-dimensional numerical integration is required, however, if there is no horizontal offset the calculation reduces to a one-dimensional numerical integration. Figure 2 shows a comparison between the theoretical predictions, calculated by this method and experimental results [11] for (nearly) ZFC for the range of gap in which the frozen-flux model is expected to be valid. The temperature of the PM was carefully controlled in the experiments. The dipole moment of the magnet was measured and assumed to be vertical in the measurements. The theoretical curve is the equivalent of a set of magnetic dipoles interacting with the set of their images. It is generally believed that this is the maximum possible force that can be generated in a PM/HTS system, yet we see that the experimental measurements exceed the theoretical calculation by as much as $20 \%$. Similar results, in which the experimental forces seem to exceed the theoretical maximum have been seen elsewhere [12]. Measurements in FC also showed a larger theoretical force than the theoretical maximum [11]. Such results motivated investigation of the role of the horizontal moment in these measurements.

\section{DIPOLE-DIPOLE FORCES}

From magnetomechanics, the force between two magnetic dipoles acting on dipole 2 is [13]

$$
F=\frac{3 \mu_{\mathrm{o}}}{4 \pi r^{5}}\left[\left(m_{1} \bullet m_{2}\right) \mathbf{r}+\left(m_{1} \bullet r\right) m_{2}+\left(m_{2} \bullet r\right) m_{1}-\frac{\left(m_{1} \bullet r\right)\left(m_{2} \bullet r\right) r}{r^{2}}\right]
$$

where $\mu_{0}$ is the permeability of free space, $m_{1}$ is the magnetic moment of the first dipole, $m_{2}$ is the magnetic moment of the second dipole, and $\boldsymbol{r}$ is the separation vector between the dipoles.

We are interested in the interaction of a dipole in the PM with its own image, as well as its interaction with the images of other dipoles. The double vector sum of the forces between each dipole and all of the images gives the total force. However, the scope of the problem can realized 
by considering the vertical force of a dipole of moment $m$, oriented at angle $\theta$ to the vertical, with its diamagnetic image, which is

$$
F_{\mathrm{z}}=\frac{3 \mu_{0} m^{2}}{4 \pi(2 z)^{4}}\left[2 \cos ^{2} \theta+\sin ^{2} \theta\right]
$$

where $\mathrm{z}$ is the height of the diple above the HTS surface. If we measure the vertical magnetic moment as $m \cos \theta$ and calculate the force assuming the moment is purely vertical, we obtain

$$
F_{\mathrm{z}}=\frac{3 \mu_{0} m^{2}}{4 \pi(2 z)^{4}}\left[2 \cos ^{2} \theta\right]
$$

If the mean angular misorientation is $\theta=20^{\circ}$, Eq. (2) will predict a force approximately $18 \%$ larger than Eq. (3), and this is consistent with the discrepancy between experimental results and theoretical predictions shown in Fig. 2. In general, a proper calculation must involve the interaction between all diples and all images and requires a knowledge of the PM magnetization as a function of location within the PM.

\section{HTS EARNSHAW ANALOG}

I now use the frozen-flux model to calculate the magnetomechanical stiffness of the dipoledipole system in the FC position. The vertical stiffness between a dipole oriented at angle $\theta$ to the vertical and its frozen image is

$$
K_{\mathrm{z}, \mathrm{fr}}=-\partial F_{\mathrm{z}, \mathrm{fr}} / \partial z=\frac{-3 \mu_{0} m^{2}}{\pi(2 z)^{5}}\left[2 \cos ^{2} \theta+\sin ^{2} \theta\right]
$$


Remembering that the diamagnetic image moves the same amount as the actual dipole and the assignment of angles shown in Fig. 1, the vertical stiffness between a diple and its diamagnetic image is opposite in sign and twice the magnitude of $\mathrm{K}_{\mathrm{z}, \mathrm{fr}}$. Thus,

$$
K_{\mathrm{z}, \mathrm{dia}}=\frac{3 \mu_{0} m^{2}}{\pi(2 z)^{5}}\left[4 \cos ^{2} \theta+2 \sin ^{2} \theta\right]
$$

The total vertical stiffness is

$$
K_{\mathrm{z}}=K_{\mathrm{z}, \mathrm{fr}}+K_{\mathrm{z}, \mathrm{dia}}=\frac{3 \mu_{0} m^{2}}{\pi(2 z)^{5}}\left[2 \cos ^{2} \theta+\sin ^{2} \theta\right]
$$

which is positive, indicating stability in the vertical direction. There is no horizontal stiffness associated with the diamagnetic image, and for the frozen image we must distinguish the $\mathrm{x}$ direction in the plane of the tilt of the magnetic dipole from the y direction which is perpendicular to the plane of the tilt. The horizontal stiffnesses are

$$
\begin{aligned}
& K_{\mathrm{x}}=\frac{3 \mu_{0} m^{2}}{\pi(2 z)^{5}}\left[\cos ^{2} \theta+\frac{3}{4} \sin ^{2} \theta\right], \\
& K_{\mathrm{y}}=\frac{3 \mu_{0} m^{2}}{\pi(2 z)^{5}}\left[\cos ^{2} \theta+\frac{1}{4} \sin ^{2} \theta\right],
\end{aligned}
$$

The horizontal stiffnesses are both positive, indicating stability in all directions, which is consistent with many experimental observations. In comparing Eqs. (6)-(8), I note the relationship

$$
K_{\mathrm{z}}=K_{\mathrm{x}}+K_{\mathrm{y}},(\mathrm{PM} / \mathrm{HTS})
$$


which is valid for any orientation of the dipole. I consider Eq. (9) to be an HTS analog to Earnshaw's theorem $[14,15]$, which when applied to magnetic systems can be written as

$$
K_{\mathrm{z}}+K_{\mathrm{x}}+K_{\mathrm{y}}=0,(\mathrm{PM} / \mathrm{PM})
$$

While the derivation was for a diple and its own images, it is readily shown that Eq. (9) is valid for any PM/HTS system with the geometry shown in Fig. 1, i.e., with a PM above an HTS with a flat surface. Between any pair of dipoles (e.g., a PM dipole and a frozen image of a second dipole), Eq. (10) must be valid. Then, using the smae logic as used in deriving Eq. (4) from Eq. (3), when we include the diamagnetic image of the second diple and constrain our system so that lal dipoles in the PM move the same incremental $\delta \mathrm{z}$, we find that $K_{\mathrm{z}}=K_{\mathrm{z}, \mathrm{fr}}+$ $K_{\mathrm{z}, \text { dia }}=-K_{\mathrm{z} \text {,ff }}$ for every interaction between any PM dipole and any frozen plus diamagnetic image from any other diple. Thus, Eq. (9) must be valid for the entire PM/HTS system. Eq. (9) has been experimentally verified for at least one system [11].

I note that Eq. (9) is strictly valid only at the FC position.

\section{CONCLUSIONS}

A generalized theorem for PM/HTS systems, analogous to Earnshaw's theorem for PM/PM systems has been derived. The theorem states that in the field-cooled position, that the vertical magnetomechanical stiffness is equal to the sum of the horizontal magnetomechanical stiffnesses.

I have shown that the presence of localized horizontal magnetic moments in PMs has the potential to obfuscate comparisons between experimental measurements and analytical or computational models of levitation forces in PM/HTS systems. A method to measure the distribution of these moments must be developed for accurate comparisons to be made. 


\section{ACKNOWLEDGMENT}

This work was supported by the U.S. Department of Energy, Energy Efficiency and Renewable Energy, as part of a program to develop electric power technology, under Contract W-31-109-Eng-38.

\section{REFERENCES}

[1] Hull J, 1999 JOM 51(11), 13

[2] Sugiura T, Hashizume H and Miya K 1991 Int. J. Appl. Electromag, Mater. 2183

[3] Tsuchimoto M, Kojima T, Takeuchi H and Honma T 1993 IEEE Trans. Magn. 293577

[4] Kuze H and Onae A 1995 J. Appl. Phys. 77770

[5] Hetland P O, Johansen T H and Bratsberg H 1996 Cryogenics 3641

[6] Navau C and Sanchez A 1998 Phys. Rev. B 58963

[7] Harris I R 1996 Rare Earth Iron Permanent Magnets, ed. J M D Coey, Oxford Science Publications, Chap. 7

[8] Assis O B G, Sinka V, and Ferrante M 1994 J. Mater. Sci. Lett. 131141

[9] Kim A S, Camp F E, and Stadelmaier H H 1994 J. Appl. Phys. 766265

[10] Kordyuk A A 1998 J. Appl. Phys. 83610

[11] Hull J R and Cansiz A 1999 J. Appl. Phys. 85 xxxx

[12] Kordyuk A A 1999 private communication

[13] Moon F C 1984 Magnetosolid Mechanics, p. 417, John Wiley \& Sons, New York

[14] Earnshaw S 1842 Trans. Cambridge Philos. Soc. 798 


\section{FIGURE CAPTIONS}

Fig. 1. Schematic of frozen-flux model. (a) Field cooling occurs at $(0, h)$ while measurement occurs at $(x, z)$. The diamagnetic image (dia) follows the permanent magnet (PM), while the frozen image (fr) remains in the initial position. (b) PM tilted at angle $\theta_{1}$ from the vertical results in diamagnetic image at angle $\pi-\theta_{1}$ and frozen image at angle $-\theta_{1}$.

Fig. 2. Levitation force versus gap between PM and HTS, comparing experimental results under zero-field cooling versus theoretical prediction using measured vertical magnetic moment of PM. 

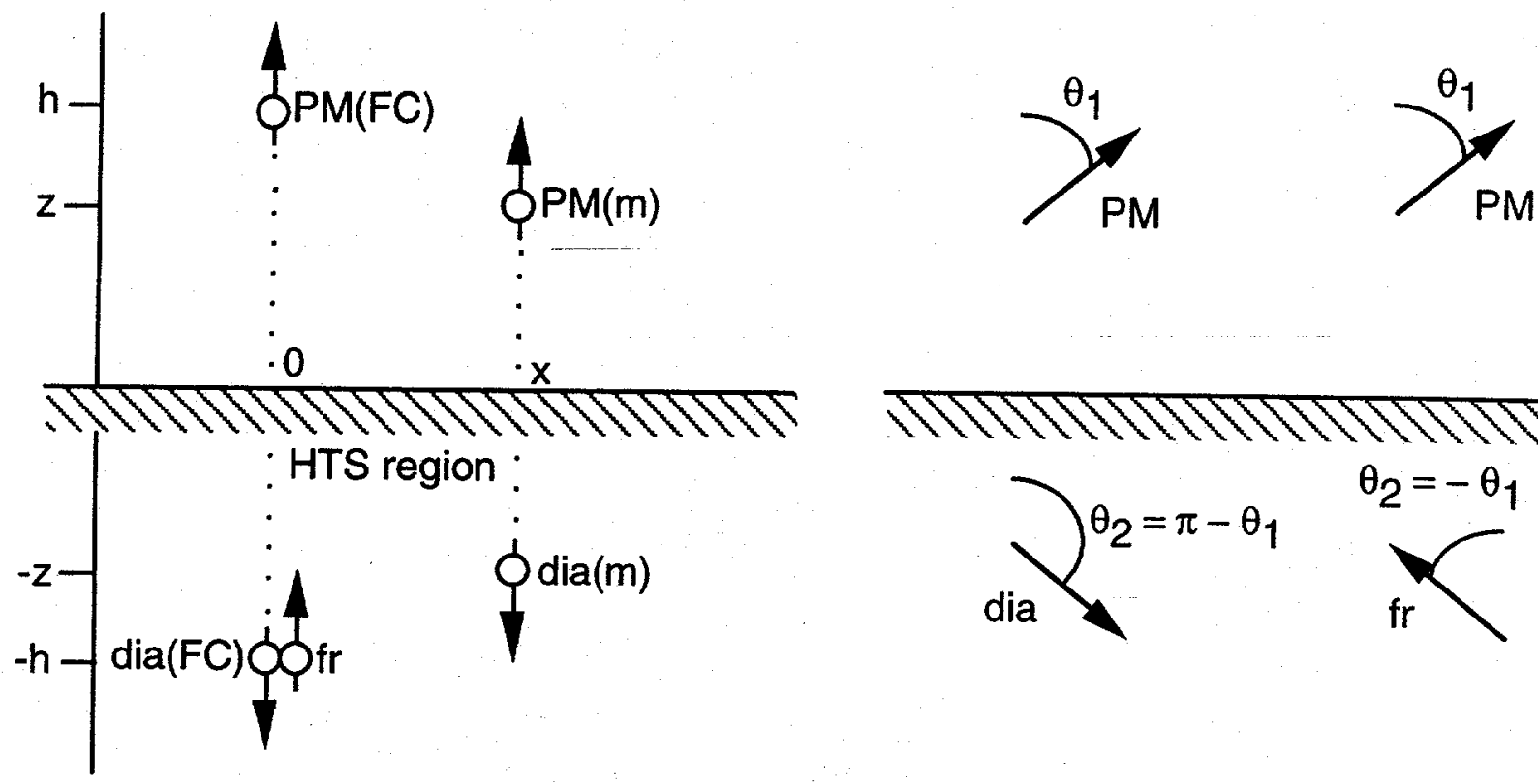

(a)

(b) 


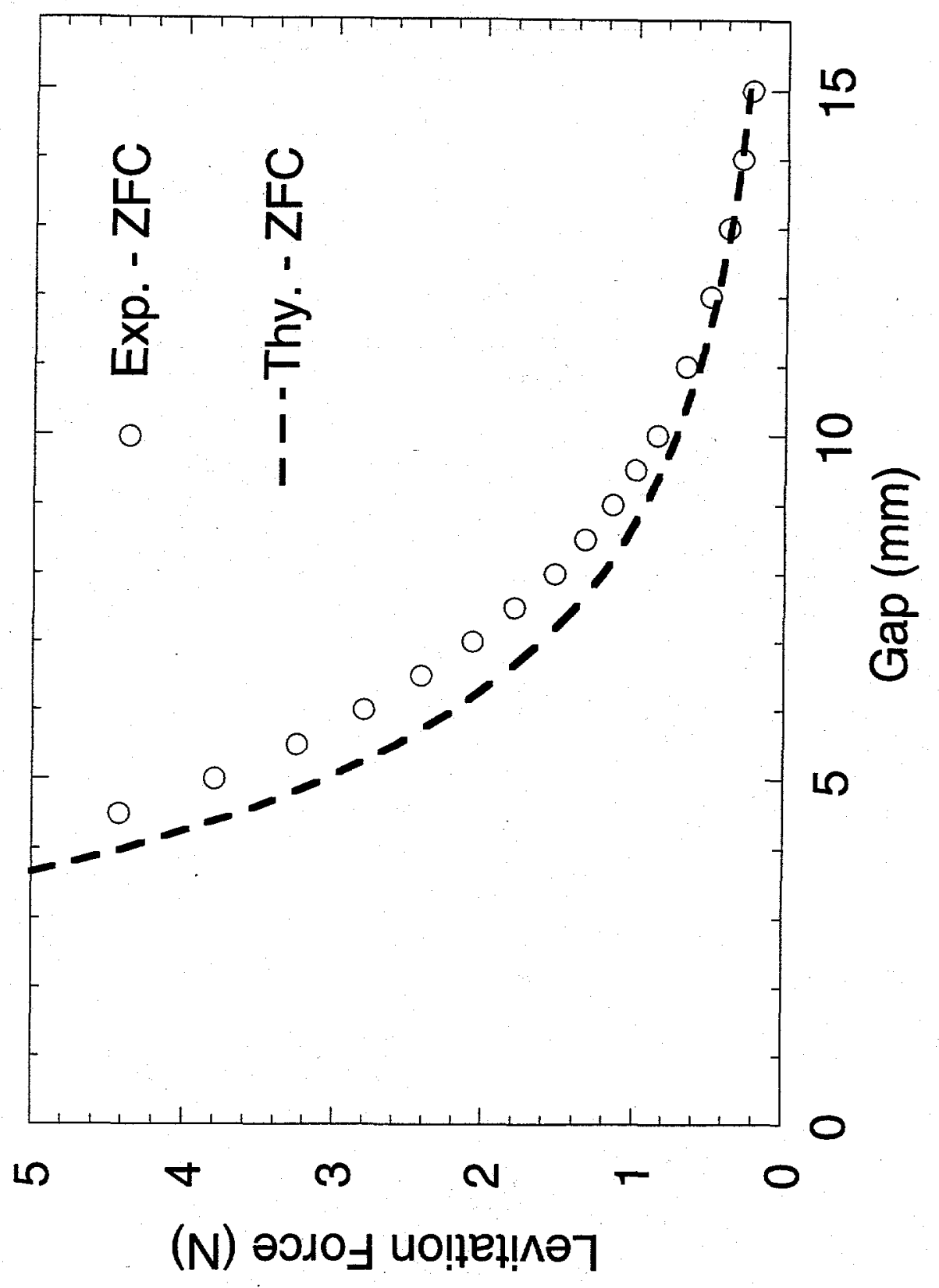

Fig. 2 